\title{
Anesthetic Concerns for Large Intracranial Cyst Excision: Expect the Unexpected!
}

\author{
Uma Hariharan ${ }^{\mathrm{a}}$, Rakesh Garg ${ }^{\mathrm{a}}$ b, Alka Gupta ${ }^{\mathrm{a}}$, Seema Wasnik ${ }^{\mathrm{a}}$
}

\begin{abstract}
The management of neurosurgical procedures for infants is always challenging for the anesthesiologists. We hereby present a case of a large ICSOL (intracranial space occupying lesion), suspected to be a hydatid cyst, which later turned out to be an infected ventricular cyst and intraoperative problems. We faced an unexpected problem of massive blood loss intraoperatively due to disease pathology. We conclude that a thorough preparation prior to operation of infected cystic lesion of the brain is required including anticipation of massive blood loss and its management. A slow decompression of the large cystic lesion should be done.
\end{abstract}

Keywords: Intracranial cyst; Massive blood loss

\section{Introduction}

Neuro-anesthesia in infants always poses a great challenge. The problems can range from difficulty in venous cannulation, securing invasive vascular lines, difficult airway, controlling intra-cranial tension, managing large fluid shifts to positioning-related issues and temperature maintenance. We hereby present a case of a large ICSOL (intracranial space occupying lesion), suspected to be a hydatid cyst, which later turned out to be an infected ventricular cyst and intraoperative problems.

\section{Manuscript accepted for publication April 29, 2011}

${ }^{a}$ Department of Anesthesiology and Intensive Care, Postgraduate Institute of Medical Education and Research and Dr Ram Manohar Lohia Hospital, New Delhi - 10001, India

bCorresponding author: Rakesh Garg, Email: drrgarg@hotmail.com

doi:10.4021/jnr20e

\section{Case Report}

A 5-month-old female baby weighing $6 \mathrm{~kg}$ was scheduled for excision of a large intracranial cystic mass lesion. On reviewing the history, child was irritable since last 4 weeks, seizures since last two weeks and progressively increasing head size. The computed tomographic scan of head revealed large hypodense lesion in left fronto-temporal-parietal region (size $7 \times 8 \mathrm{~cm}$ ) which was thick walled and compressing foramen of Monro (Fig. 1). There was dilatation of contralateral lateral ventricle with multiple satellite lesions and perilesional edema, midline shift to right of $2.6 \mathrm{~cm}$ and subfalcine herniations. Magnetic resonance imaging (MRI) revealed well-defined heterogenous area of altered signal intensity involving entire left fronto-temporal-parietal region, with associated peri-lesional edema and mass effect. A differential diagnosis of cystic lesion and hydatid cyst was made.

Presently, child was on valproate sodium and dexamethasone. Her GCS score was 8 . Her pulse rate was 160 beats/ $\mathrm{min}$. Her hematological and biochemical investigations were normal. In the operating room, electrocardiogram, noninvasive blood pressure and pulse oximeter were attached. General anesthesia was induced with intravenous fentanyl $(15 \mu \mathrm{g})$ and thiopentone $(50 \mathrm{mg})$, and neuromuscular blockade was achieved with vecuronium $(0.6 \mathrm{mg})$. Trachea was intubated with $5.5 \mathrm{~mm}$ ID uncuffed tube and bilateral equal air entry was confirmed. Temperature and end tidal carbon dioxide monitoring was also initiated. Right femoral venous cannulation and left femoral artery cannulation were done. Anesthesia was maintained with isoflurane in oxygen and nitrous oxide (50:50) (MAC 1). The mannitol was administered before opening of the dura and on skull retraction, dura was tense and bulging. A large cyst was seen arising from the frontal horn of lateral ventricle with extensions into deep brain matter. A total of $300 \mathrm{~mL}$ of thick, light greenish colored pus was slowly aspirated and cyst was later removed in toto, which left behind a large lacuna in the brain. During cyst excision, there was severe bleeding of around $800 \mathrm{~mL}$ over a short time-period which was replaced with $550 \mathrm{~mL}$ of packed red blood cells, $100 \mathrm{~mL}$ of fresh frozen plasma and $50 \mathrm{~mL}$ of platelet concentrate. A total of $750 \mathrm{~mL}$ of crys- 


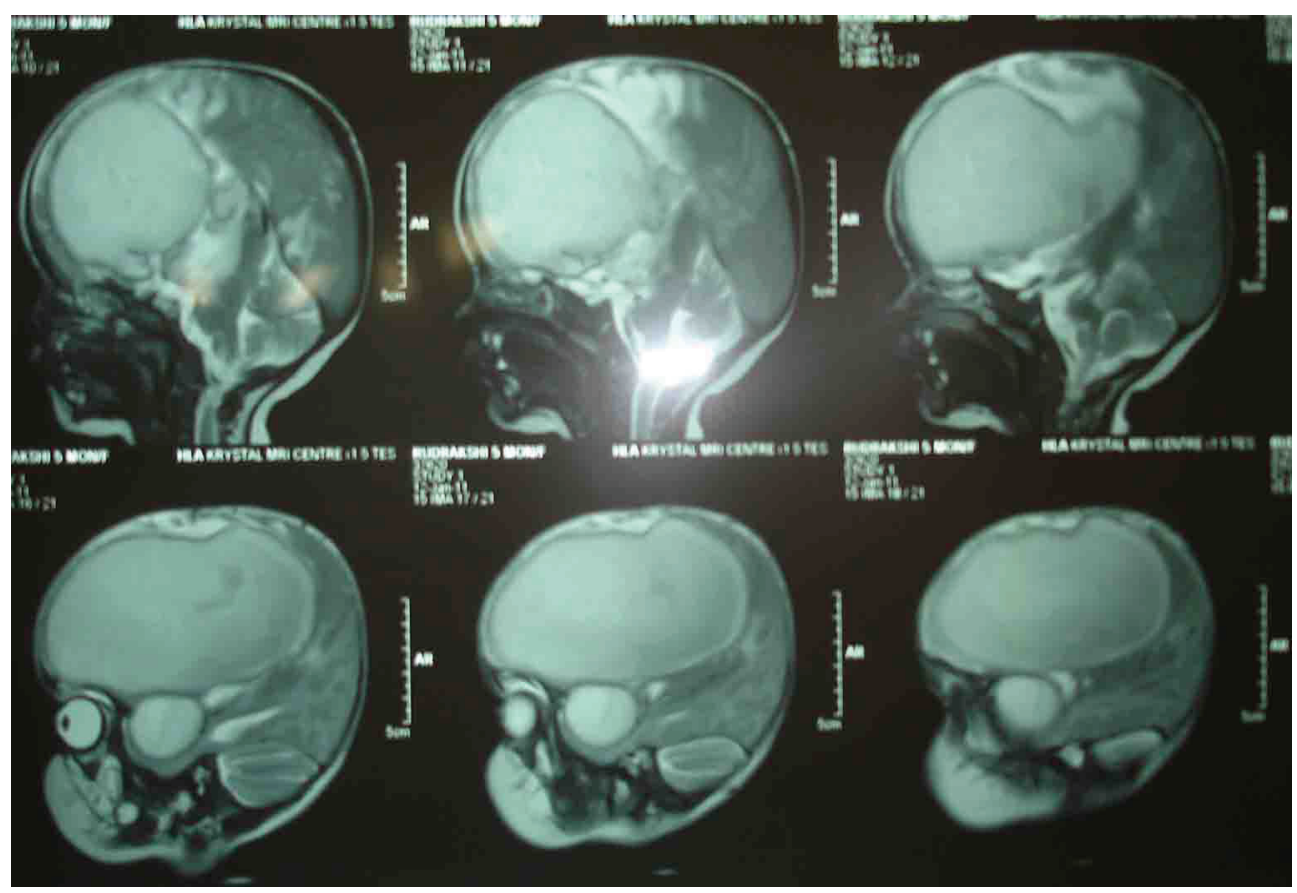

Figure 1. Preoperative head CT scan.

talloids were given and the child passed $550 \mathrm{~mL}$ of urine overall. The vitals were maintained throughout, except for a brief period of hypotension during the bleeding episode (corrected with blood transfusion). Primary water-tight dural closure was done after cyst excision. The child was shifted to the pediatric ICU for elective ventilation and further management. Postoperative analgesia was provided with rectal paracetamol. Initially, the child started passing lots of urine $(7.7 \mathrm{~mL} / \mathrm{kg} /$ hour) (simulating a diabetes insipidus like picture), which got spontaneously corrected after the first 24 hours. All vital parameters were maintained and the child was successfully extubated after 72 hours. The Glasgow coma scale (GCS) improved to 12 . On examination of cyst fluid and cyst, gram stain and AFB were negative, plenty of pus cells were seen and no hydatid elements or trophozoites of ente-amoeba were noted. On bacterial culture, Catalase negative Streptococcus species were isolated. Smears from the cyst showed acute and chronic inflammatory cells against necrotic background. Child was managed accordingly and was discharged uneventfully after 12 days.

\section{Discussion}

Patients with intracranial cysts usually present with focal neurological deficit and features of raised intracranial pressure; the latter may be due to the large size or due to interference with pathway of CSF flow [1]. Surgically intact cyst excision is the ideal treatment [2]. The challenge in such cases arises not only because of pediatric age group but also due to neurosurgical procedure. Such patients pose many intraoperative challenges, especially when the cyst is found to be infected. The first concern is the risk of the spillage of the pus in the adjoining tissues and its consequences. So careful dissection is required during the removal of the cyst. In our case, the neurosurgeons carefully aspirated the cyst contents and then dissected the cyst to avoid spillage of the pus. Secondly, the infected tissue leads to neovascularization of the surrounding tissues and also increased vascularity. So during the dissection, there is additional risk of increased bleeding in such cases as happened in our case. Normally, the distended cyst, keeps the vessels under pressure but after opening the dura, such pressure effect is reduced and this is further reduced after the excision. This increases the blood flow to these fragile tissues and thus increases chances of bleeding. Similar problems happened in our case requiring massive blood transfusion in our patient. So sufficient blood products must be ensured in such patient prior to operation.

Also, a lacunae exists after the removal of large cyst in the brain. This could lead to traction of the cerebral structures and may lead to onset of neurological deficits in the postoperative period. Our patient had features of diabetes insipidus but subsided with conservative management.

We conclude that a thorough preparation prior to operation of infected cystic lesion of the brain is required including anticipation of massive blood loss and its management. A slow decompression of the large cystic lesion should be done. 


\section{References}

1. Onal C, Erguvan-Onal R, Yakinci C, Karayol A, Atambay M, Daldal N. Can the requirement of a diver- sion procedure be predicted after an uncomplicated intracranial hydatid cyst surgery? Pediatr Neurosurg 2006;42(6):383-386.

2. Ali M, Mahmood K, Khan P. Hydatid cysts of the brain. J Ayub Med Coll Abbottabad 2009;21(3):152-154. 